UCRL-JC-121677

PREPRINT

\title{
Local Positioning System
}

\author{
Ron Kyker
}

This paper was prepared for submittal to the IEEE Conference Proceedings, WESCON

San Francisco, CA

November 8, 1995

July 25, 1995

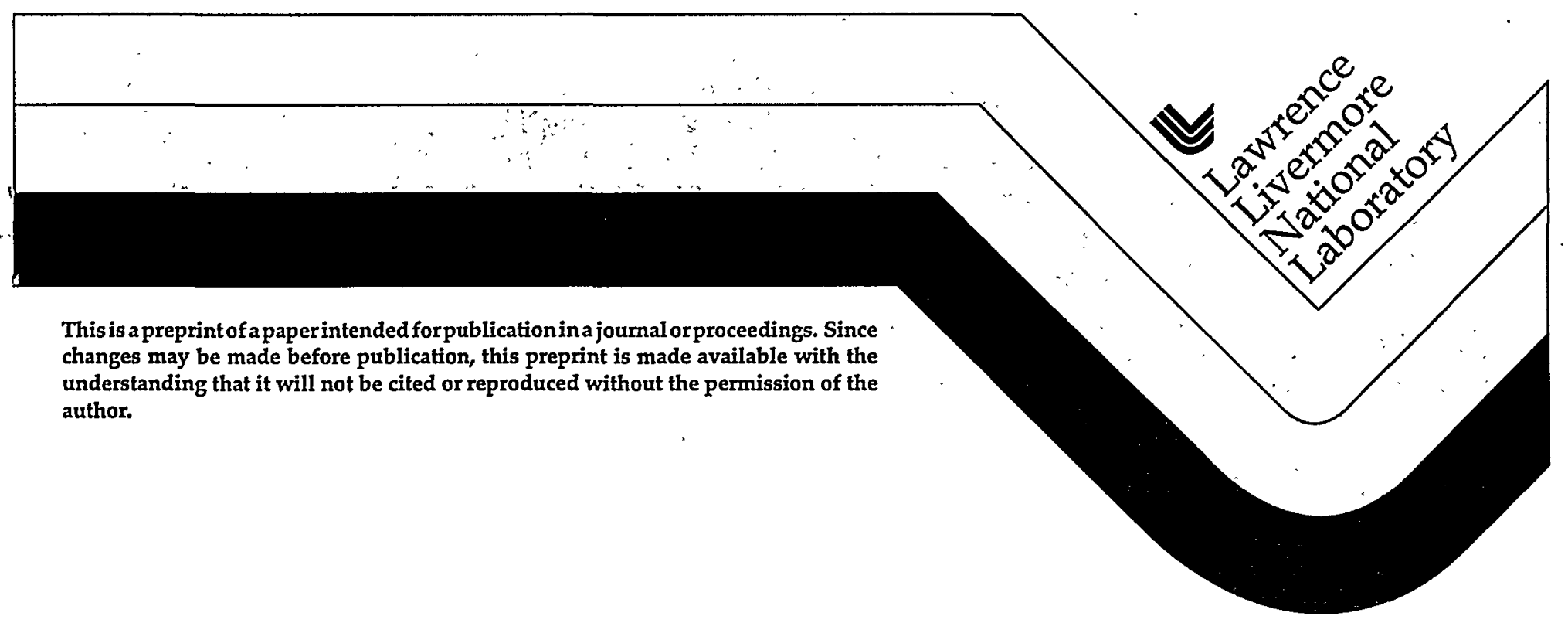




\section{DISCLAIMER}

This document was prepared as an account of work sponsored by an agency of the United States Government. Neither the United States Government nor the University of California nor any of their employees, makes any warranty, express or implied, or assumes any legal liability or responsibility for the accuracy, completeness, or usefulness of any information, apparatus, product, or process disclosed, or represents that its use would not infringe privately owned rights. Reference herein to any specific commercial product, process, or service by trade name, trademark, manufacturer, or otherwise, does not necessarily constitute or imply its endorsement, recommendation, or favoring by the United States Government or the University of California. The views and opinions of authors expressed herein do not necessarily state or reflect those of the United States Government or the University of California, and shall not be used for advertising or product endorsement purposes.

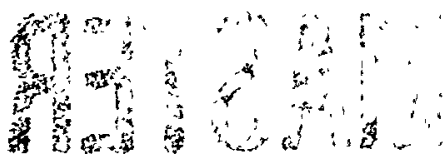




\title{
Local Positioning System
}

\author{
By Ron Kyker \\ IEEE Student Member No. 03476538 \\ Lawrence Livermore National Laboratory \\ 7000 East Ave. L-493 \\ Livermore, Ca 94550 \\ (510) $422-5453$
}




\section{Table of Contents}

1.0 Abstract

$2.0 \quad$ Introduction

3.0 The System Design

3.1 Distance Sensor Design

3.2 Compass Design

\subsubsection{Compass Sensor}

\subsubsection{Compass Assembly Design}

3.3 Microcontroller Assembly Design

3.4 Software Design

5.0 Test Results

5.1 Compass Sensor Testing

5.2 Heading Accuracy Tests

5.3 LPS System Tests

5.3.1 Locational Accuracy Tests

6.0 Conclusions

7.0 References

Appendix A: The Software Flow Diagram

Appendix B: System Specifications 


\subsection{Abstract}

Navigation systems have been vital to transportation ever since man took to the air and sea. Early navigation systems utilized the sextant to navigate by starlight as well as the magnetic needle compass. As electronics and communication technologies improved, inertial navigation systems were developed for use in ships and missile delivery. These systems consisted of electronic compasses, gyro-compasses, accelerometers, and various other sensors. Recently, systems such as LORAN and the Global Positioning System (GPS) have utilized the properties of radio wave propagation to triangulate position.

The Local Positioning System (LPS), described in this paper, is an implementation of a limited inertial navigation system designed to be used on a bicycle. LPS displays a cyclist's current position relative to a starting location. This information is displayed in Cartesian-like coordinates. To accomplish this, LPS relies upon two sensors, an electronic compass sensor and a distance sensor. The compass sensor provides directional information while the distance sensor provides the distance traveled. This information yields a distance vector for each point in time which when summed produces the cyclist's current position. LPS is microprocessor controlled and is designed for a range of less than 90 miles.

* Work performed under the auspices of the U.S. Department of Energy by Lawrence Livermore National Laboratory under Contract No. W-7405-Eng-48 


\subsection{Introduction}

The objective of LPS is to design a system that displays a cyclist's current position relative to a starting location. The system consists of three separate units: the distance sensor, the electronic compass sensor assembly, and the microcontroller assembly. A block diagram is shown below in Figure 1.0. The distance sensor mounts to the front wheel fork. and produces a pulse on every revolution of the wheel. The compass sensor assembly mounts behind the seat post and above the rear tire and transmits directional data to the microcontroller. The microcontroller assembly mounts on the handlebars for ease of operation and visibility, much like a standard cycle computer. The microcontroller assembly interfaces to the distance sensor and compass sensor assembly providing computation resources and data display. The specifications are listed in Appendix B.

Figure 1.0

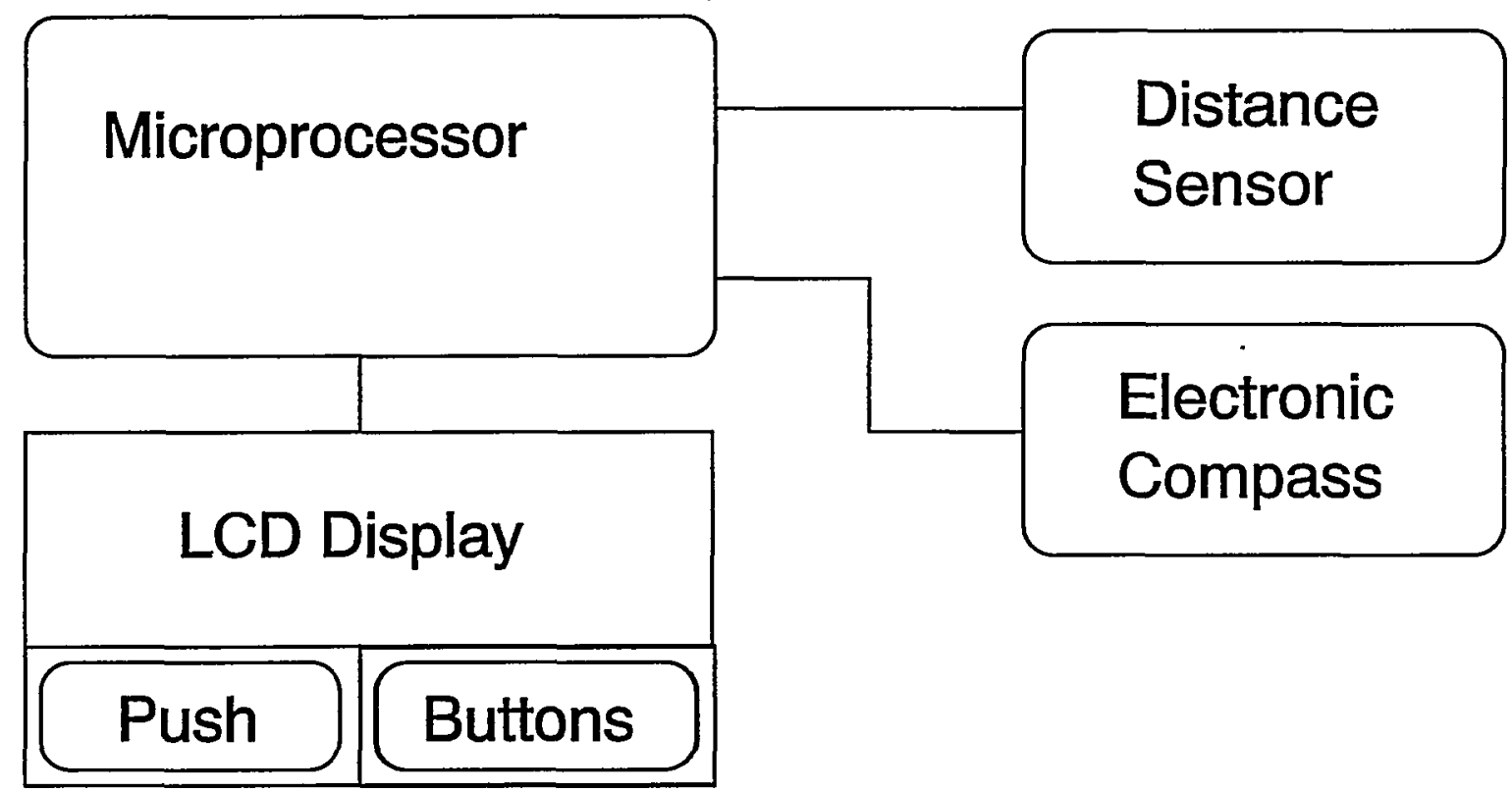




\subsection{The System Design}

Several requirements of the design are evident from the LPS concept and objectives stated above. First, a distance or speed sensor is required in order to determine the distance the vehicle has traveled. Second, a digital compass or other bearing sensor is required to show which direction the vehicle is traveling. Finally, a CPU is required to perform necessary computations.

\subsection{Distance Sensor Design}

Several options were available for implementing a distance sensor. A Hall-effect switch and magnet combination proved to be the superior choice since optical sensors are difficult to mount for this application and reed switches have switch bounce. The Halleffect switch provided a clean signal with no switch bounce and easy sensor mounting.

The distance sensor is shown in Figure 2.0. The distance sensor consists of a permanent magnet and a Hall-effect switch. The magnet is fastened to the bicycle spokes while the sensor is mounted to the bicycle fork. The Hall-effect switch triggers with a magnetic field strength as small as 160 Gauss. This translates to a magnet clearance of about $1 / 4$ inch with the magnet used. The switch requires a 5 VDC supply and an external resistor. The rise time for this device is very fast and it produces a clean bounceless pulse on every revolution of the wheel when the magnet passes by the sensor.

The distance sensor output is connected to the microcontroller IRQ maskable interrupt line. The IRQ line is programmed for edge triggering to catch the rising edge of the pulse provided by the wheel sensor. 


\section{Distance Sensor}
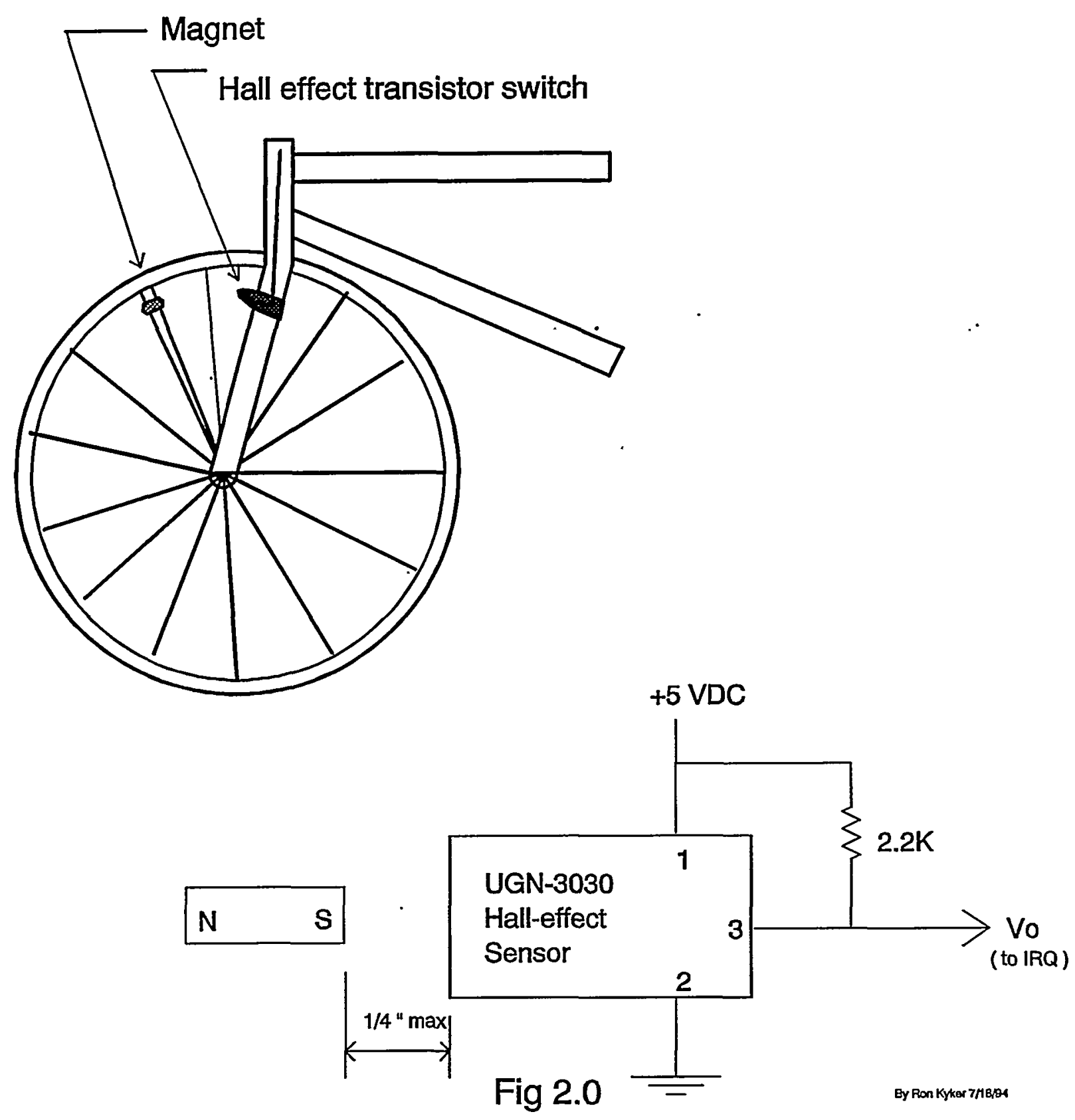


\subsection{Compass Design}

Current compass sensors are based upon several technologies. These inelude: fluxgate magnetometer, magneto-resistive, and Hall-effect sensors. Since magnetometer and magneto-resistive compasses are complex and expensive, a Hall-effect based sensor was selected. This required more hardware but lowered overall costs.

\subsubsection{Compass Sensor}

The Hall effect compass sensor consists of a commercially packaged magnetic needle compass with two Hall-effect transistors positioned perpendicular to one another outside the compass needle. The Hall-effect transistors measure the strength and polarity of the magnetic needle of the compass. When the North end of the compass needle is pointing towards the sensor a maximum voltage output is produced. Likewise with the South end, a minimum voltage is produced. This method allows the direction of the earth's magnetic field to be measured indirectly since the earth's magnetic field is too weak to be directly by currently Hall devices. When rotated 360 degrees, the sensor produces two analog waveforms similar to sine curves, one 90 degrees out of phase of the other. 
The compass sensor outputs can be approximately described mathematically by

$$
\text { Output1 }=2.5+.4 \operatorname{Sine}(\Theta h)
$$

and

$$
\text { Output } 2=2.5+.4 \operatorname{Sine}(\Theta \mathrm{h}+90)
$$

where $\Theta h$ is the magnetic heading angle from $0-360$ degrees. The signal output voltage is approximately 800 millivolts peak to peak with a 2.5 volt DC offset. This yields an output signal varying from about 2.1 to 2.9 volts.

The compass output signals are digitized and converted to a numeric heading. For maximum accuracy, heading information is extracted from the portion of the sine . curves with the greatest slope. A steep slope gives the greatest voltage change per degree heading change. This produces the greatest heading accuracy. Given the phase difference between the two waveforms, one of the two outputs will have a steep slope at any point in time.

Serial analog to digital (A/D) converters are used to digitize the sensor output signal as close to the source as possible. This minimizes noise affects. Two voltage. reference pots are connected to the serial A/D's and are used to set the minimum and maximum digitizing voltages. This calibration process is necessary due to manufacturing variations in each sensor. Since the sensor supplies an $800 \mathrm{mv}$ peak-peak signal, direct digitization can be accomplished, with no amplification required. 


\subsubsection{Compass Assembly Design}

Figure 3.0 shows the digital compass design. The main components include the Hall effect based analog compass sensor, a voltage regulator which supplies closely regulated 5 VDC power, and two serial $A / D$ converters. Local regulation is necessary to produce a noise-free, stable 5 VDC supply for isolation from the microprocessor supply. The compass sensor voltage regulator employs a power-off mode which is used to power down the compass assembly when it is not being read by the MCU. Since the compass sensor consumes $20 \mathrm{ma}$, powering down results in significant power conservation. A complete power-up and compass read cycle requires about $7 \mathrm{~ms}$ to complete. Using this design feature, current consumption was reduced to 2 ma when 16 readings are taken per second.

The digital compass operates as follows: First the microcontroller (MCU) powers up the voltage regulator on the compass assembly. After a power up delay, the MCU signals the A/D's to digitize the two waveforms. Then the MCU clocks out the serial data and reassembles it into two bytes, one for each waveform. The compass-tomicroprocessor interface requires only six wires. 


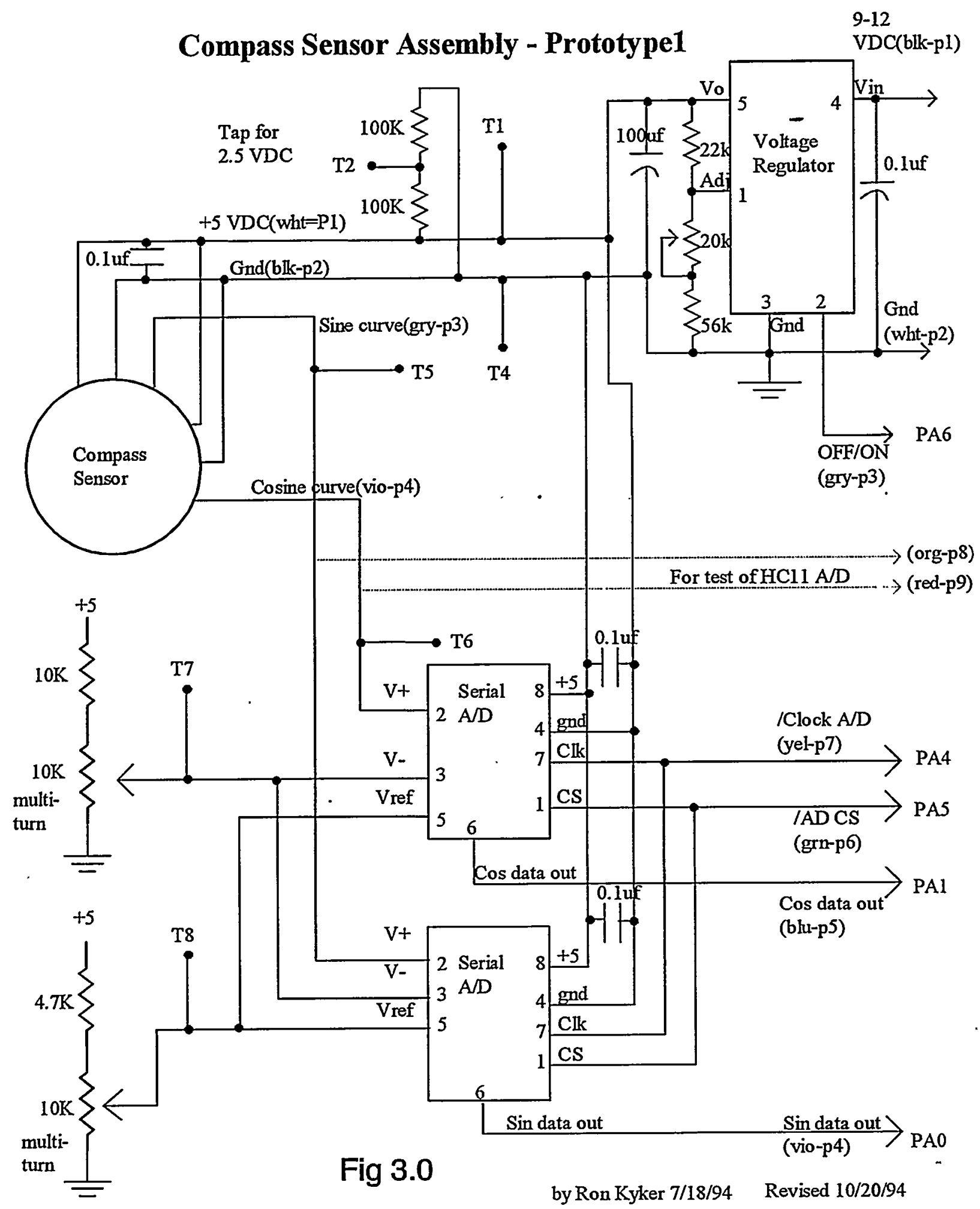




\subsection{Microcontroller Assembly Design}

The microcontroller design requirements included: low power, limited $\mathrm{I} / \mathrm{O}$, and a development platform which was readily available and easy to implement. Many processors sufficed, however, the 68HC11 Micro-Controller Unit (MCU) was chosen because of the available development environment, documentation, and evaluation units called the EVBUs are offered at a discount to students.

Figure 4.0 shows a overview of the microcontroller interface design. The compass assembly and two push-button switches for user input are connected to port $A$ of the HC11 EVBU. The switches are debounced using a NAND logic gate and pull-up resistors. The switch debouncing could have been accomplished via software; however to alleviate additional software and time constraints; a hardware solution was chosen.

An LCD display provides data display while an external non-volatile RAM (NVRAM) provides data an program storage for non-volatile operation. These are interfaced directly to the address and data bus: Control signals for these devices are provided by a Programmable Logic Device (PLD). A latch provides address and data demultiplexing since the HC11 multiplexes the lower 8 address/data lines. The NVRAM is an $8 \mathrm{~K} \times 8$ non-volatile RAM/shadow ROM which saves the RAM contents to ROM on power down and restores the RAM contents from the ROM on power-up. This was useful for software development since it allowed easy program downloading to non-volatile RAM. Power is provided by an 8 cell AA battery pack. Individual power regulators for the MPU and compass assemblies establish separate power sources which isolate noise effects. 


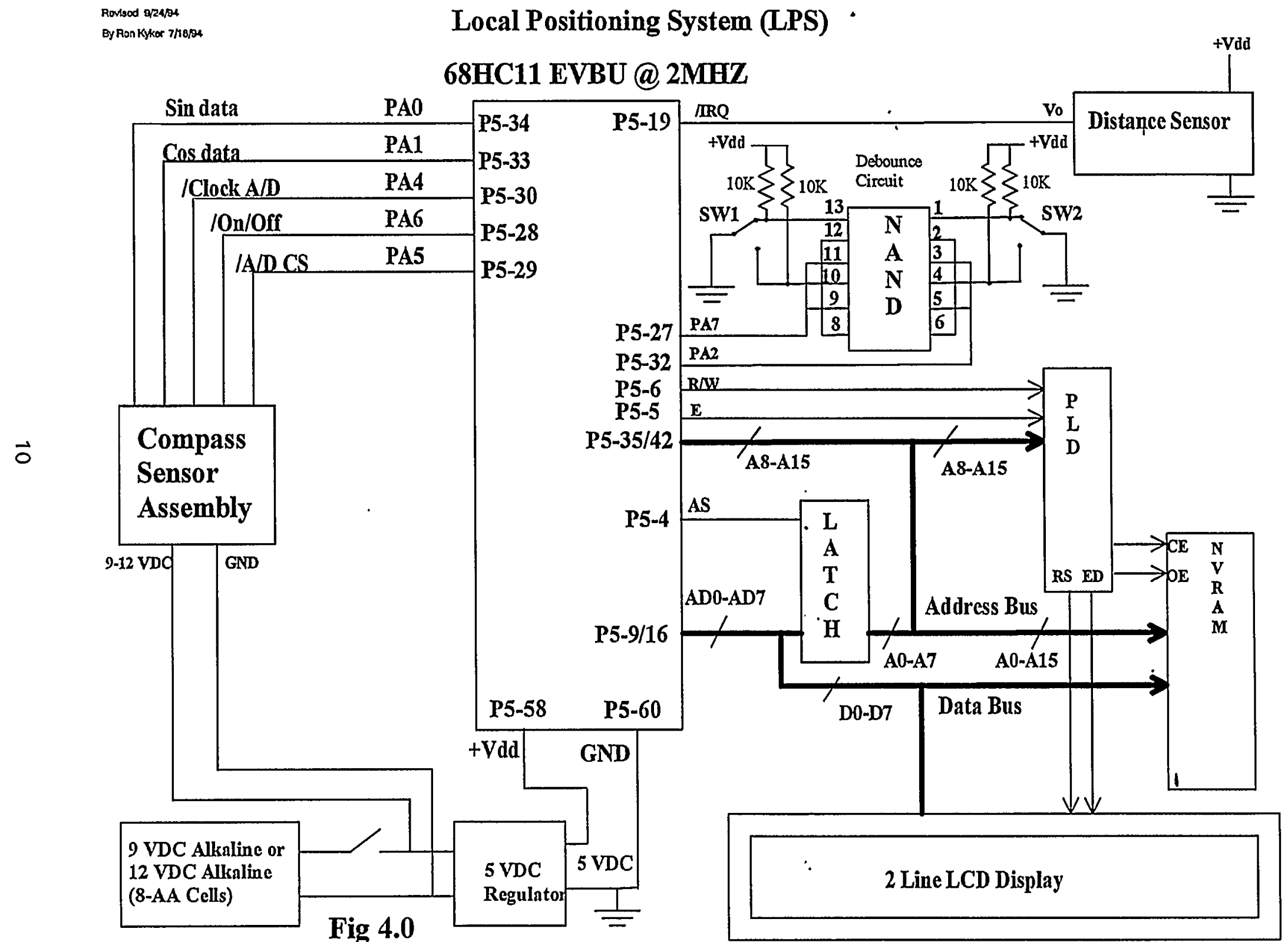




\subsection{Software Design}

The basic flow diagram for the LPS system software is shown in Appendix A. The software includes interrupt service routines for the distance/wheel sensor (IRQ) and a realtime interrupt routine (RTI) that is used as an event timer to time intervals for reading the compass sensor. The software was written in assembly and uses floating point math . routines supplied by Motorola to execute most mathematical computations. Floating point routines are required due to the large variation in numeric values with time and accuracy requirements. The main software written for this system assembles to 2345 Bytes.

Look Up Tables (LUT) are used to translate digitized curve data from the compass to degrees. These tables were produced from a mathematical model using a spreadsheet program. While less accurate than a manual calibration process, the model is reproducible and gives adequate results. 


\subsection{Test Results}

Several tests were executed during and after development. Initial testing was executed to determine effects and operation of the compass sensor. Final testing focused on performance of the complete system.

\subsection{Compass Sensor Testing}

Early in the design, it was determined that variations existed in each compass sensor, causing deviations from the ideal sinewave curves. The sensor produced sine curves of $.812 \mathrm{Vp}$-p and $.704 \mathrm{Vp}$-p respectively with centers at $2.54 \mathrm{~V}$ and 2.543 respectively. This variation requires that each sensor be calibrated to allow digitizing from peak to peak only.

An additional problem, sensor tilt error, was discovered during testing. The sensor is extremely sensitive to tilt, contrary to the manufacturers specification. Data samples were taken for various tilt angles and recorded in Table I below showing large errors for small tilt angles.

Variations in compass sensor with tilt.

Table I

\begin{tabular}{|l|l|l|l|l|}
\hline Tilt Angle, de & Starting Output & Tilted Output & Voltage Differenc & $\begin{array}{l}\text { Heading Error, } \\
\text { degrees }\end{array}$ \\
\hline$\sim 5$ & 2.576 & 2.628 & $52 \mathrm{mv}$ & 7.5 \\
\hline$\sim 10$ & 2.576 & 2.7 & $124 \mathrm{mv}$ & 18 \\
\hline$\sim 15$ & 2.576 & 2.745 & $169 \mathrm{mv}$ & 25 \\
\hline$\sim 30$ & 2.579 & 2.815 & $236 \mathrm{mv}$ & 36 \\
\hline
\end{tabular}


Compass curve data samples are translated using mathematically generated LookUp Tables instead of manually created ones. Producing manually created tables would have required that samples be taken for each of 360 degrees, a labor intensive process. Compass readings were taken at various headings. The error in heading reading for various samples is recorded below in Table II below.

Compass error using mathematically generated Look-Up Tables (LUT).

Table II

\begin{tabular}{|l|l|}
\hline Actual Heading & Compass Error (degrees) \\
\hline 0 & +1 \\
\hline 45 & +1 \\
\hline 90 & +2 \\
\hline 135 & $-5 *$ \\
\hline 180 & +2 \\
\hline 225 & +3 \\
\hline 270 & +2 \\
\hline 315 & 0 \\
\hline
\end{tabular}

This method proved to be a more accurate than originally speculated. In the future, a calibration process could be used to offset the above values such that errors fell on both sides thereby improving accuracy. The -5 measurement above is believed to be in error since it does not correlate with any other measurements. With the errors reported above; the system is accurate enough to meet the specification. 


\subsection{Heading Accuracy Tests}

The above compass accuracy is important only in the respect that it contributes greatly to positional accuracy. Given that a compass has an error of a certain number of degrees, Table III below shows the error in position this would produce. A heading of 45 degrees is assumed. The error was computed by dividing the error vector magnitude by the actual position vector magnitude which is computed for a 100 mile distance.

Error in position given a constant error in heading.

Table III

\begin{tabular}{|l|l|l|}
\hline Error (degrees) & Reading & Magnitude of error \\
\hline 1 & 46 & $1.8 \%$ \\
\hline 2 & 47 & $2.3 \%$ \\
\hline 4 & 49 & $6.7 \%$ \\
\hline 6 & 51 & $10.6 \%$ \\
\hline 8 & 53 & $14.2 \%$ \\
\hline 10 & 55 & $17.5 \%$ \\
\hline
\end{tabular}

\subsection{LPS System Tests}

Two trips were made for the system test. The second was made after it was discovered that the compass sensor had rotated during the test creating additional error. A fixture was used for the second run to prevent sensor movement. For both tests, a 2 mile loop was made to and from the University of the Pacific campus. Measurements were taken at several intersections which appeared on a Stockton, CA map. This was used to check the positional accuracy against the map. All testing was performed on reasonably flat ground. Testing on flat ground provided a means to test the effects of normal tilt in the roll axis during riding while minimizing the error due to pitch. 


\subsubsection{Locational Accuracy Tests}

For the locational accuracy test, the relative coordinates from the LPS system were recorded at several intersections. These were compared to measurements taken from a map which was measured as accurately as possible. Errors for trial \#1 will increase near the end due to the shift in the compass sensor mentioned earlier. The suspect measurement is marked with a *. Relative coordinates are shown in Table $\mathrm{V}$ below for different intersections.

Locational accuracy test trials performed in Stockton; CA near University of the Pacific:

Table V

\begin{tabular}{|l|l|l|l|l|l|l|l|}
\hline Location & Map X & Map Y & Magnitude & LPS X & LPS Y & Magnitude & Error \% \\
\hline $\begin{array}{l}\text { CC \& } \\
\text { Pershing }\end{array}$ & $.01 \mathrm{~N}$ & $.43 \mathrm{~W}$ & .43 & $.01 \mathrm{~N}$ & $.45 \mathrm{~W}$ & .45 & $4.7 \%$ \\
\hline $\begin{array}{l}\text { Pershing \& } \\
\text { Larry Heller }\end{array}$ & $.43 \mathrm{~N}$ & $.58 \mathrm{~W}$ & .72 & $.42 \mathrm{~N}$ & $.60 \mathrm{~W}$ & .73 & $1.4 \%$ \\
\hline Burns Tower & $.78 \mathrm{~N}$ & $.06 \mathrm{E}$ & .78 & $.80 \mathrm{~N}$ & $.11 \mathrm{E}$ & .81 & $3.8 \%$ \\
\hline $\begin{array}{l}\text { Stadium \& } \\
\text { Kensington }\end{array}$ & $.66 \mathrm{~N}$ & $.16 \mathrm{~W}$ & .68 & $.67 \mathrm{~N}$ & $.26 \mathrm{~W}$ & $.52 *$ & $-23.5 \% *$ \\
\hline $\begin{array}{l}\text { Stadium \& } \\
\text { Kensington }\end{array}$ & $.66 \mathrm{~N}$ & $.16 \mathrm{~W}$ & .68 & $.68 \mathrm{~N}$ & $.13 \mathrm{~W}$ & .69 & $1.5 \%$ \\
\hline
\end{tabular}




\subsection{Conclusions}

The Local Positioning System (LPS) performed according to the designed specifications. LPS provides a method of determining relative position. Several engineering problems exist with the current design, most regarding the compass sensor.

- These problems have been thoroughly documented along with proposed solutions which have not been fully tested and implemented due to limited time. The most serious problems involve compass sensor sensitivities. These include: isolating the compass sensor from magnetic aberrations, sensitivities of the compass sensor due to tilt, temperature, and other environmental conditions, and finally calibration considerations since no two sensors are identical.

It is believed that the above problems can be solved.. Some of the proposed solutions appear promising. Alternate designs have be documented which will reduce circuitry by $75 \%$ and provide an auto calibration mechanism. Miniaturization will follow. The final design would be similar to a standard cycle computer and would provide the same functionality in addition to LPS functions. LPS would however include the compass sensor assembly which will mount under the seat. Parts retail for around $\$ 50$ retail. 


\subsection{References}

Noble, Richard, "Electronic Fluxgate Compass"

Electronics World \& Wireless World, Jan 1992 P14-19

Sokolowski, Steve"A Digital Compass"

Modern Electronics August 1990 P22-25

Ota, Kazuhide and Hashimoto, Toshio

" Development of Electronic Compass for Automobile Using Magnetoresistive Elements" Automotive display systems and IVHS, Society of Automotive Engineers 1993 P81-89

Liu, Sheng-wu and Zhang, Zhao-nian

"A High Accuracy magnetic Heading System Composed of Fluxgate Magnetometers and a Microcomputer"

Northwestern Polytechnical University, Xian, China ; thesis 1985

Garner, Doug "Smart Compass" R\&D magazine, October 1986

Available from NASA/Langley Research Center, Hampton, Va. 
Appendix A

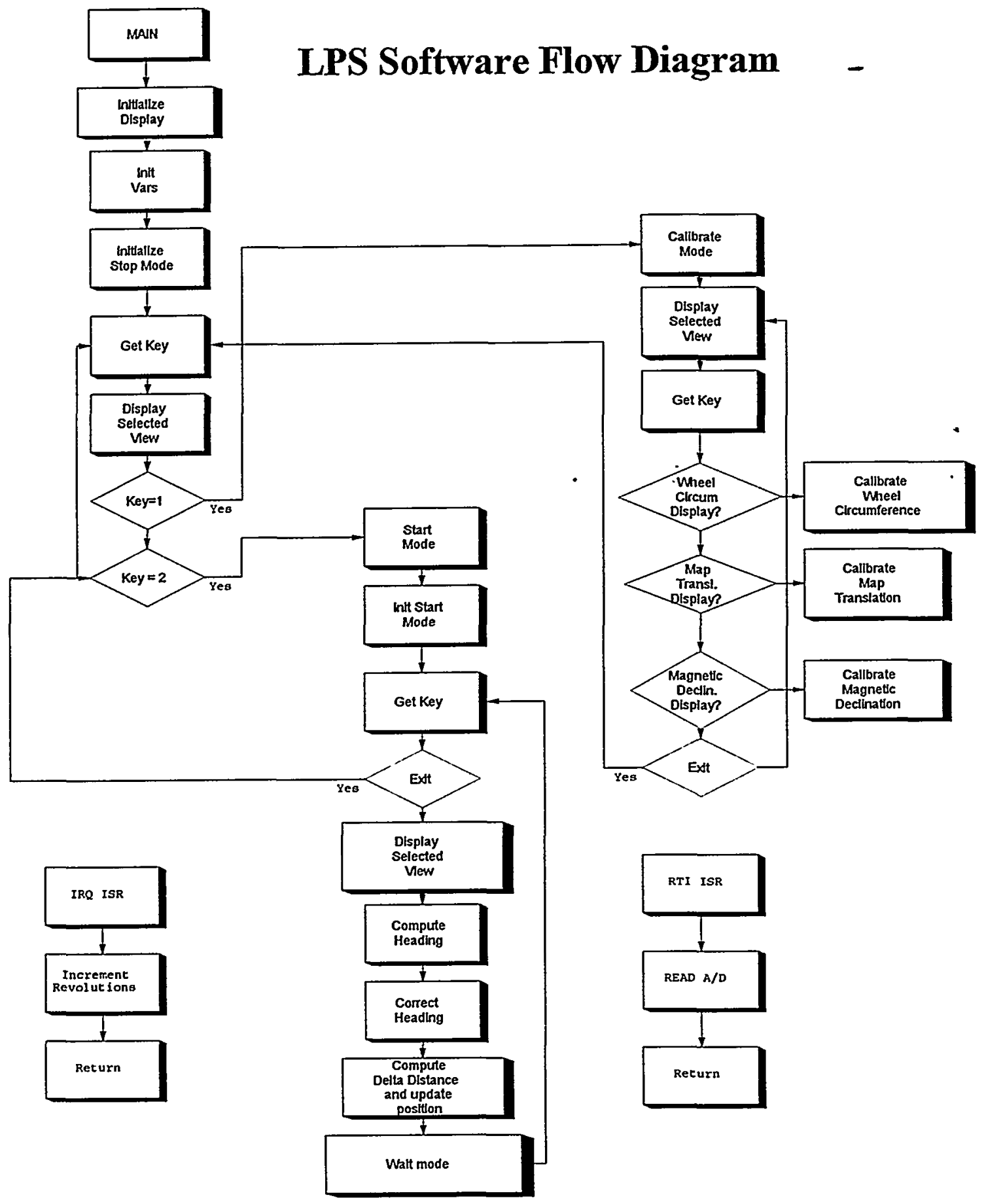




\section{Appendix B}

System Specifications

Location accuracy error: $\quad<10 \%$ for range of $1-100$ miles

Heading accuracy error: $\quad+/-5.0$ degrees out of 360 degrees

Physical dimensions (max): $\quad$ Sensor Assembly: $\quad 3$ " $\times 4$ " $\times 4$ "

CPU Assembly: $\quad 4^{\prime \prime} \times 4^{\prime \prime} \times 1.5$

Operation time: $\quad$ minimum of 12 hours

Suggested retail price: $\quad<\$ 100$

Temperature Range: $\quad 20-70$ degrees Celsius

Power requirements: $\quad<20$ ma from 9 VDC Alkaline cell or 8 AA cells 
About the Author

Ron Kyker He graduated from DeVry Institute of Technology in 1984 with a Bachelor of Science degree in Electronics Engineering. Technology and moved to Livermore, $\mathrm{Ca}$ to work for Lawrence Livermore National Laboratory (LLNL) where he has worked for the past 10 years. In 1993 he won the LLNL Undergraduate Scholarship which pays full tuition and salary for the recipient to complete the last two years of undergraduate work. Ron has been working on his Bachelor of Science degree in Electrical Engineering at University of the Pacific in Stockton, California. He currently holds a 3.98 GPA in Engineering classes, is a member of IEEE, Tau Beta Pi, and Eta Kappa Nu. Ron enjoys bicycling, fishing, music, model airplanes, and of course electronics. If accepted, he plans to attend Stanford University for graduate work while working at LLNL. He is undecided on doctorate work. 



$$
-1
$$

